\title{
SIMULATION IN NURSING EDUCATION: REVIEW OF RESEARCH
}

\author{
Sulochana Ghimire, ${ }^{1}$ Anuja Kachapati
}

\begin{abstract}
\section{INTRODUCTION}

Nursing education consists of the theoretical and practical training provided to nurses with the purpose to prepare them for their duties as nursing care professionals. The scope of nursing practice reflects all the role and responsibilities undertaken by the nurse to address the full range of human experiences and responses to health and illness. The instructional strategies utilized in both didactic and clinical components of nursing education courses are highly influential in determining critical thinking and clinical decision making ability as well as in developing the psychomotor skill performance of new graduates. Simulation provides nursing students with opportunities to practice their clinical and decision-making skills through various real-life situational experiences. Although endorsed in nursing curricula, its effectiveness is largely unknown.
\end{abstract}

KEYWORDS Medical simulation, Nursing education, Nursing simulation.

Universal College of Nursing Sciences, UCMS, Bhairahawa, Nepal

DOI: http//doi.org/10.3126/jucms.v8i02.34308

For Correspondence

Ms. Sulochana Ghimire

Universal College of Nursing Sciences

UCMS, Siddharthanagar-1, Rupandehi, Nepal

Email:sanguinegal12@gmail.com 


\section{INTRODUCTION}

Caring is a central element of nursing practice. The American Nurses Association (ANA) stated that an essential feature of professional nursing is the provision of a caring relationship that facilitates health and healing. ${ }^{1}$

Nursing education focuses on educating health care people about effective ways to deliver the health care to patients. The aim of nursing education is a development of the nursing profession. ${ }^{2}$ Nursing requires complex combinations of knowledge, performance, skills and attitudes to provide care to the patients. ${ }^{3}$

Simulation has been defined as a situation in which a particular set of conditions is created artificially in order to study or experience something that is possible in real life; or a generic term that refers to the artificial representation of a real world process to achieve educational goals via experimental learning. ${ }^{4}$ Simulations are instructional scenarios where the learner is placed in a "world" defined by the teacher. They represent a reality within which students interact. The teacher controls the parameters of this "world" and uses it to achieve the desired instructional results. Students experience the reality of the scenario and gather meaning from it. A simulation is a form of experiential learning. It is a strategy that fits well with the principles of student-centered and constructivist learning and teaching. The inventiveness and commitment of the participants usually determines the success of a simulation. ${ }^{5}$ It is a cheaper, faster, and efficient way for practice and learning that helps people understand how they should act in real life situations. ${ }^{6}$ Simulation helps in developing self-confidence among students by helping students to link between theory and practical. It provides an opportunity to study and analyze critical teaching problems. As a result of role playing, it helps in the development of critical thinking in students and teachers. ${ }^{7}$

Over the past two decades, there has been an exponential and enthusiastic adoption of simulation in healthcare education Internationally. Simulation-based healthcare education has great potential for use throughout the healthcare education continuum, from undergraduate to continuing education. It can also be used to train a variety of healthcare providers in different disciplines from novices to experts. ${ }^{8}$ Although medical simulation is relatively new, simulation has been used for a long time in other high risk professions such as aviation. Medical simulation allows the acquisition of clinical skills through deliberate practice rather than an apprentice style of learning. Simulation tools serve as an alternative to real patients. A trainee can make mistakes and learn from them without the fear of harming the patient. ${ }^{9}$ Inter-professional simulation provide health profession program educators with an effective means to prepare future practitioners to engage in meaningful collaboration. ${ }^{10}$ Improving patient safety requires a multifaceted approach. Training alone cannot do the job; it must be accompanied by improvements to clinical systems and processes, better design of equipment and user interfaces, and the creation and maintenance of a true culture of safety in the workplace. If these other things are not achieved, no amount of training can overcome the barriers to optimal safety. CRM (Crisis Resource Management) or teamwork training, especially with simulation, can be a 'lever for culture change' because it is intrinsically clinically engaging. ${ }^{11}$

The relationship between theory and practice is a complex challenge in professional education. This is widely documented and commonly termed as a "gap". To reduce this gap, theoretical knowledge and practical experience must be integrated. SBL is a pedagogical approach that can be considered a "third learning space" between coursework and practicums; this approach may bring the content and process of theoretical work and practical training closer to each other. ${ }^{12}$ A systematic review of research on technology enhanced simulation in health professions revealed that in comparison with no intervention, technology-enhanced simulation training in health professions education is consistently associated with large effects for outcomes of knowledge, skills, and behaviors and moderate effects for patient-related outcomes. ${ }^{13}$ Simulation is a method of teaching that allows or requires learners to apply theory to practice in an integrated manner. If the simulation demonstrates more than a single event or parameter at a time, nurses learn to identify relationships essential and common to clinical practice. ${ }^{14}$

Development of and research into SBME (Simulation Based Medical Education) have grown and matured over the past 40 years on substantive and methodological grounds. Simulation-based medical education is a complex service intervention that needs to be planned and practiced with attention to organizational contexts. ${ }^{15}$ The simulated operating theatre could serve as an environment for the development of surgical competence among surgical trainees. Objective, structured, and multimodal assessment of performance during simulated procedures could serve as a basis for focused feedback during training of technical and team skills. ${ }^{16}$

Simulation learning is routinely used in medical education and training, and it has been used in nursing education for quite a while. It is used to promote clinical competency and reflective-thinking skills. Simulation as a remediation allows students to practice skills, increase confidence, and minimize the likelihood of error prior to working in a clinical setting. The safety of patients depends on the accuracy of those providing care, and simulation as a learning method provides 
an opportunity to attain a level of experience with no human involvement. ${ }^{17}$ The debriefing step at the conclusion of any simulation activity allows trainees to clarify and consolidate the insights and lessons learned from the experience. As well as task training, skills training, and procedure training, simulation can be used to provide error response and group training for the high-reliability teams working in the operating room, emergency room, and intensive care unit. Globally, simulation is fast being incorporated into professional assessment and programs for maintenance of certification. Such programs may become more efficient with the use of crowdsourcing to evaluate video-recorded performance of procedures. $^{18}$

The old teaching method of "see one, do one and teach one" which has long been used to teach nursing skills and to promote the acquisition of clinical expertise is no longer accepted as the best way to teach students. A nurse's confidence in her/his ability is essential for good patient care. Only when nursing students have confidence in their own abilities, they are able to shift focus to the needs of their patients. Shifting from their own needs to that of a patient is essential to being a safe and competent practitioner. ${ }^{19}$

A qualitative study conducted to explore the students' experiences regarding high-fidelity simulation revealed that debriefing is one of the more important components of the experience. Reflection during debriefing allows the student to critically think through the lived experience. Although simulated experience will never be exactly the same as patient care, it allows the student to experience a critical event before they are responsible for one in a working environment ${ }^{20}$ The meta-analysis conducted to explore the effectiveness of simulation-based nursing education depending on fidelity showed that simulation-based nursing education was effective in various learning domains, with a pooled random-effects standardized mean difference of 0.70 . These results suggest that simulation-based nursing educational interventions have strong educational effects, with particularly large effects in the psychomotor domain. ${ }^{21}$

The systematic review conducted on the impact of simulationbased CRM (Crisis Resource Management) teaching on transfer of learning to the workplace and subsequent changes in patient outcomes revealed that CRM skills learned at the simulation center are transferred to clinical settings, and the acquired CRM skills may translate to improved patient outcomes, including a decrease in mortality. ${ }^{22}$ Students' knowledge scores were compared before and after an educational intervention during SBL (Simulation Based Learning) in a quasi-experimental study conducted on impact of a new pedagogical intervention on nursing students' knowledge acquisition in SBL. Results revealed that the intervention group had significantly higher scores on a knowledge test conducted after the simulations in comparison to the scores in the control group. In both groups scores were highest on knowledge of symptoms and lowest on knowledge of pathophysiology; the intervention group had significantly higher scores than the control group on both topics. ${ }^{23}$

A quasi-experimental, prepost-test study conducted on 285 students in a pre-licensure clinical nursing course revealed that the simulation learning experience provides students with knowledge and skill gains and challenges them with memorable experiential learning that they can apply to clinical practice. ${ }^{24}$ A study conducted on Predictors of Knowledge Gains Using Simulation in the Education of Pre-licensure Nursing Students revealed that HPS (Human Patient Simulation) can independently improve test scores thereby providing evidence that HPS; is an effective teaching methodology for pre-licensure nursing students regardless of age, learning style, or critical thinking ability. ${ }^{25}$ A pre-post-test study conducted to determine whether self-reported confidence and knowledge measures are indicators of clinical performance observed in a simulation-based assessment. Results showed that the intervention group had a significantly higher post-test mean score than the control group for knowledge and clinical performances. ${ }^{26}$

In a study conducted with stroke unit nurses, high-fidelity simulation was incorporated into stroke unit education for registered nurses during their orientation to the acute stroke unit at a large tertiary care center. Nurses participated in three scenarios for successful care for stroke unit patients. Overall evaluation by nursing staff after the simulation and debriefing was very positive; $100 \%$ of the participants ranked the effectiveness of the simulations as excellent. ${ }^{27} \mathrm{~A}$ study conducted on simulator-based medical education service revealed that initial evaluations among both preclinical and clinical students suggest that simulation is highly accepted and increasingly demanded. For some learners, simulation may allow complex information to be understood and retained more efficiently than can occur with traditional methods. Moreover, the study suggests that simulation can be integrated into existing curricula of almost any medical school or teaching hospital in an efficient and cost-effective manner. ${ }^{28}$

In areas of medical training, simulation has been demonstrated to lead to improvements in medical knowledge, comfort in procedures, and improvements in performance during retesting in simulated scenarios. Simulation has also been shown to be a reliable tool for assessing learners and for teaching topics such as teamwork and communication. Only a few studies have shown direct improvements in clinical 
outcomes from the use of simulation for training. ${ }^{29}$ A systematic review of the literature examining the effectiveness of simulation as a teaching tool that aimed to evaluate current literature on the use of clinical simulation in health care education. The findings revealed that there is gap in the literature pertaining to evaluating the effectiveness of using simulations as a teaching tool, and support the need for further research into the evaluation of simulation as a teaching tool. $^{30}$

\section{CONCLUSION}

Simulation is a generic term that refers to an artificial representation of a real world process to achieve educational goals through experiential learning. Medical simulation has been found to enhance clinical competence at the undergraduate and postgraduate levels. Simulation is an effective teaching and learning method when best practice guidelines are adhered to. Simulation may have some advantage over other teaching methods, depending on the context, topic and method. Simulation allows the opportunity to learn and practice critical care skills in a controlled and safe environment. The research study on simulation in nursing education is limited at this time. The research available is also not sufficient to support having simulation replace clinical education, but simulation is a wonderful bridge between theory and practice.

\section{REFERENCES}

1. Meyer G, Lavin MA. Vigilance: The essence of nursing. Online Journal of Issues in Nursing. 2005 Jun 23;10(3):38-51.

2. Olsson HM, Gullberg MT. Nursing education and definition of the professional nurse role. Expectations and knowledge of the nurse role. Nurse Education Today. 1991 Feb 1;11(1):30-6.

3. Cowan DT, Norman I, Coopamah VP. Competence in nursing practice: A controversial concepta focused review of literature. Nurse education today. 2005 Jul 1;25(5):355-62.

4. Flanagan B, Nestel D, Joseph M. Making patient safety the focus: Crisis resource management in the undergraduate curriculum. Medical Education. 2004 Jan;38(1):56-66.

5. What is simulation? 2018 July 26. https://teaching.unsw.edu. $\mathrm{au} /$ simulations

6. Rimon G. 4 Benefits of Learning Simulations. 2016 November 22:8-10.

7. http://www.studylecturenotes.com/curriculum-instructions /advantages-and-disadvantages-of-simulated-teachingmethod
8. Motola I, Devine LA, Chung HS, Sullivan JE, Issenberg SB. Simulation in healthcare education: A best evidence practical guide. AMEE Guide No. 82. Medical Teacher. 2013 Oct 1;35(10):e1511-30.

9. Al-Elq AH. Simulation-based medical teaching and learning. Journal of Family and Community Medicine. 2010 Jan; 17(1):35.

10. Murdoch NL, Bottorff JL, McCullough D. Simulation education approaches to enhance collaborative healthcare: A best practices review. International Journal of Nursing Education Scholarship. 2014 Jan 8;10(1):307-21.

11. Gaba, DM. Crisis resource management and teamwork training in anaesthesia. British Journal of Anaesthesia. 2010 July 01;105(1):3-6.

12. Hatlevik IK. The theory practice relationship: Reflective skills and theoretical knowledge as key factors in bridging the gap between theory and practice in initial nursing education. Journal of Advanced Nursing. 2012 Apr 1;68(4):868-77.

13. Cook DA, Hatala R, Brydges R, Zendejas B, Szostek JH, Wang AT, Erwin PJ, Hamstra SJ. Technology-enhanced simulation for health professions education: A systematic review and meta-analysis. JAMA. 2011 Sep 7;306(9):978-88.

14. Rauen CA. Simulation as a teaching strategy for nursing education and orientation in cardiac surgery. Critical Care Nurse. 2004 Jun 1;24(3):46-51.

15. McGaghie WC, Issenberg SB, Petrusa ER, Scalese RJ. A critical review of simulation based medical education research: 2003-2009. Medical Education. 2010 Jan;44(1):50-63.

16. Moorthy K, Munz Y, Adams S, Pandey V, Darzi A. A human factors analysis of technical and team skills among surgical trainees during procedural simulations in a simulated operating theatre. Annals of Surgery. 2005 Nov;242 (5):631.

17. Ward-Smith P. The effect of simulation learning as a quality initiative. Urologic Nursing. 2008 Dec 1;28(6):471.

18. McDougall EM. Simulation in education for health care professionals. British Columbia Medical Journal. 2015 Dec 1;57(10): 444-448.

19. Leigh GT. High-fidelity patient simulation and nursing students' self-efficacy: A review of the literature. International Journal of Nursing Education Scholarship. 2008 Sep 26;5(1):17.

20. Lasater K. High-fidelity simulation and the development of clinical judgment: Students' experiences. Journal of Nursing Education. 2007 Jun 1;46(6): 269-276.

21. Kim J, Park JH, Shin S. Effectiveness of simulation-based nursing education depending on fidelity: A meta-analysis. BMC Medical Education. 2016 Dec;16(1):152.

22. Boet S, Bould MD, Fung L, Qosa H, Perrier L, Tavares W, 
Reeves S, Tricco AC. Transfer of learning and patient outcome in simulated crisis resource management: A systematic review. Canadian Journal of Anesthesia/Journal canadiend'anesthésie. 2014 Jun 1;61 (6):571-82.

23. Haukedal TA, Reierson I $\AA$, Hedeman H, Bjørk IT. The impact of a new pedagogical intervention on nursing students' knowledge acquisition in simulation-based learning: A quasiexperimental study. Nursing Research and Practice. 2018;2018.

24. DeBourgh GA, Prion SK. Using simulation to teach prelicensure nursing students to minimize patient risk and harm. Clinical Simulation in Nursing. 2011 Mar 1;7(2):e47-56.

25. Shinnick MA, Woo M, Evangelista LS. Predictors of knowledge gains using simulation in the education of prelicensure nursing students. Journal of Professional Nursing. 2012 Jan 1;28(1):41-7.

26. Liaw SY, Scherpbier A, Rethans JJ, Klainin-Yobas P. Assessment for simulation learning outcomes: A comparison of knowledge and self-reported confidence with observed clinical performance. Nurse Education Today. 2012 Aug 1;32(6):e35-9.

27. Aebersold M, Kocan MJ, Tschannen D, Michaels J. Use of simulation in stroke unit education. Journal of Neuroscience Nursing. 2011 Dec 1;43(6):349-53.

28. Gordon JA, Oriol NE, Cooper JB. Bringing good teaching cases "to life": A simulator-based medical education service. Academic Medicine. 2004 Jan 1;79(1):23-7.

29. Okuda Y, Bryson EO, DeMaria Jr S, Jacobson L, Quinones J, Shen B, Levine AI. The utility of simulation in medical education: What is the evidence? Mount Sinai Journal of Medicine. 2009 Aug 1;76(4):330-43.

30. Harder BN. Use of simulation in teaching and learning in health sciences: A systematic review. Journal of Nursing Education. 2010 Jan 1;49(1):23-8. 